\title{
Estrategias de lucha contra la pobreza en América Latina. Análisis crítico de los planteamientos de algunos organismos internacionales
}

\author{
Julio Boltvinik*
}

En este trabajo se analizan de manera crítica los enfoques que sobre la medición de la pobreza y sobre las políticas de lucha contra la misma, han presentado organismos como el Banco Mundial (BM) y la Comisión Económica para América Latina (CEPAL), los cuales se contrastan algunas veces con aquellos correspondientes al Proyecto Regional para la Superación de la Pobreza del Programa de Naciones Unidas para el Desarrollo (PNUD). El articulo comienza por, clasificar las diferentes formas de medir la pobreza de los citados organismos, las cuales conducen a la presentación de cifras bastante disímbolas sobre su incidencia en América Latina. En cuanto al análisis de las políticas de lucha contra la pobreza, el autor ubica primero el sentido de las mismas para cada organismo (políticas inmersas en el modelo neoliberal en el caso del BM; en el "enfoque integrado" de (EPAL y en el "desarrollo sin pobreza" del PNUD); a continuación se refiere a la manera como los tres organismos explican la existencia y agudización de la pobreza, para presentar más adelante, de forma bastante detallada, los objetivos y compc :tentes de las estrategias (reducción sostenible de la pobreza para el $B M$; crecimiento con equidad, ambientalmente sustentable y en democracia, para la CEPAL; aumento de la calidad y cantidad de la vida de los miembros de la sociedad, para el PNUD). El trabajo finaliza con un balance comparativo crítico de los rasgos básicos, sobre todo de las propuestas del $B M$ y de la CEPAL, mostrando tanto sus diferencias como sus puntos de convergencia.

\section{Introducción}

Este trabajo analiza críticamente dos enfoques institucionales sobre la pobreza en América Latina: el de cepal y el del Banco Mundial. Además en algunas partes del texto ellos se contrastan con el enfoque del Proyecto Regional para la Superación de la Pobreza del PNud (de aquí en adelante PRSP-PNUD) ${ }^{1}$

De entrada es necesario señalar que los tres enfoques institucionales difieren en diversos aspectos importantes: xico.

* Profesor-investigador del Centro de Estudios Sociológicos de El Colegio de Mé-

1 La propuesta de CEPAL está contenida en tres trabajos, precedidos por el lema de “Transformación Productiva con Equidad" (CEPAL, 1990, 1992; CEPAL-UnesCo, 1992). Las estrategias de lucha contra la pobreza que viene impulsando el Banco Mundial están descritas en diversos documentos (вм, 1990, 1993a, 1993b, 1993c y 1993d). La propuesta del PRSP-PNUD se encuentra en Desarrollo sin pobreza (PRSP-PNLD, 1990, 1992a). 
a) El grado de adopción institucional de la propuesta. Mientras los del Banco Mundial y la CEPAL han sido adoptados al más alto nivel institucional y han generado culturas institucionales en su entorno, el del PRSP-PNUD no ha sido apropiado siquiera por la Oficina para América Latina de este organismo, a pesar de que fue formalmente aprobado por los representantes de los gobiernos del subcontinente en la II Conferencia Regional para la Superación de la Pobreza, celebrada en Quito en 1990 (PRSP-PNUD, 1990).

b) El grado en que las propuestas están siendo aplicadas. Mientras las ideas del Banco Mundial se ponen en práctica mediante los créditos de la institución, las propuestas de los otros dos organismos sólo se llevan a la práctica en la medida en que logran convencer a los gobiernos respectivos y en que éstos dispongan de fondos no condicionados.

c) El grado en el que los planteamientos buscan una coherencia explicita entre conceptuación y medición por una parte y políticas por la otra. Mientras es muy grande en el PRSP-PNUD, es bastante más laxo en los otros dos casos; en el caso de cEPAL incluso no están plenamente integrados en un único documento. ${ }^{2}$

\section{La medición de la pobreza}

\section{Antecedentes metodológicos}

Los métodos de línea de pobreza (LP) y de necesidades básicas insatisfechas (NBI), son los predominantes en América Latina y los que aplican -solos o combinados- los tres estudios. El de tP consiste en comparar el ingreso (o el consumo) per cápita, o por adulto equivalente de un hogar, con la llamada línea de pobreza, expresada en los mismos términos. Los hogares con ingresos menores que la línea de pobreza se consideran pobres, y la misma característica se atribuye a cada una de las personas que en ellos habitan. El punto clave de este

${ }^{2}$ Los aspectos de medición y sus conceptualizaciones subyacentes que aquí se analizan muy resumidamente, pueden encontrarse en dos trabajos de cEPAL (lo que llamaré el trabajo CEPAL-PNUD, 1990, y su antecedente, al que llamaré el trabajo CEPAL-70), uno del Banco Mundial referido a la distribución del ingreso y la pobreza en América Latina (1993) y el del PRSP-PNUD (1990, 1991, 1992 y 1992a). Las fuentes para conocer los resultados del primer trabajo son CEPAl-PNUD (1990 y 1992), así como Beccaria, Boltvinik, Fresneda, Sen et al. (1992). Del segundo, principalmente O. Altimir (1979). 
método consiste en la forma como se define la línea de pobreza. En Estados Unidos y América Latina ha predominado la variante que consiste en definir una canasta normativa alimentaria (CNA), calcular su costo y multiplicar éste por el recíproco del coeficiente de Engel (porcentaje del gasto que se dedica a alimentos) de algún grupo de hogares, para obtener la línea de pobreza. A este procedimiento le he llamado la variante de canasta normativa alimentaria del método de LP y he mostrado que lo que mide es la pobreza alimentaria -la incapacidad del hogar de satisfacer la necesidad alimentaria- y no la pobreza en general.

El método tradicional de necesidades básicas insatisfechas (NBI) consiste en comparar la situación de cada hogar, en cuanto a un grupo de necesidades específicas, con una serie de normas que, para cada una de ellas, expresan el nivel mínimo por abajo del cual se considera insatisfecha la necesidad. Los hogares que tienen una o más NBI se consideran pobres, lo mismo que todos sus miembros. Los puntos críticos de este método son la selección de necesidades, la definición del umbral mínimo para cada una de ellas, y el criterio de definición de pobreza (v.g. si es suficiente una NBI para definir como pobre al hogar). En la práctica latinoamericana, el método se ha visto restringido fuertemente por la información disponible a nivel de hogar, proveniente de censos y encuestas. Los indicadores utilizados han sido los referidos a hacinamiento; viviendas inadecuadas (por sus materiales) o improvisadas; abastecimiento inadecuado de agua; carencia (o inadecuación) de sistemas para la eliminación de excretas; inasistencia a la escuela primaria de los menores; y un indicador indirecto de los ingresos del hogar, que asocia el nivel educativo del jefe de éste con la tasa de dependencia económica del mismo. Nótese que indicadores disponibles generalmente en censos y encuestas, como el nivel educativo de los miembros del hogar o el acceso a la electricidad, no son tomados en cuenta.

Para el análisis crítico de estos métodos, se postula que la satisfacción de las necesidades básicas de una persona o de un hogar, depende de las siguientes seis fuentes de bienestar: $a$ ) el ingreso corriente; b) los derechos de acceso a servicios o bienes gubernamentales de carácter gratuito (o subsidiados); c) la propiedad, o derechos de uso, de activos que proporcionan servicios de consumo básico (patrimonio básico acumulado); d) los niveles educativos, las habilidades y destrezas, entendidos no como medios de obtención de ingresos, sino como expresiones de la capacidad de entender y hacer; $e$ ) el tiempo disponible para 
la educación, la recreación, el descanso, y para las labores domésticas, y f) los activos no básicos y la capacidad de endeudamiento del hogar.

Entre algunas de estas fuentes de bienestar existe la posibilidad de sustitución. Con un mayor ingreso se pueden sustituir algunos derechos de acceso, atendiendo necesidades como salud y educación privadamente, o sustituir la no propiedad de algunos activos de consumo (por ejemplo, rentar una vivienda). Esta sustituibilidad no es perfecta, sin embargo. Con ingresos adicionales no se puede sustituir la falta de tiempo disponible para educación y recreación; si no están desarrolladas las redes básicas de agua y drenaje, no será posible (o será muy costoso) acceder a estos servicios por la vía privada.

La limitación principal de los métodos de línea de pobreza y de necesidades básicas insatisfechas (tal como éste se ha venido aplicando en América Latina) consiste en que proceden, el primero, como si la satisfacción de necesidades básicas dependiera solamente del ingreso o del consumo privado corriente de los hogares; el segundo, en sus aplicaciones usuales (haciendo caso omiso del último indicador), elige indicadores de satisfacción de necesidades que básicamente dependen de la propiedad de activos de consumo (vivienda) o de los derechos de acceso a servicios gubernamentales (agua, eliminación de excretas y educación primaria), por lo cual implícitamente deja de tomar en cuenta las demás fuentes de bienestar. Es decir, ambos tienen una visión parcial de la pobreza y en la medida en que las fuentes de bienestar consideradas por ambos métodos son distintas, de inmediato podemos concluir que no son procedimientos alternativos, como se les suele considerar, sino complementarios. Además, el método de NBI, tal como se aplica actualmente, tiene una característica muy dañina. Ésta consiste en que el número de pobres identificados no es independiente del número de rubros de necesidades básicas seleccionados. Mientras mayor es éste, mayor es la incidencia de la pobreza.

Métodos de medición de la pobreza utilizados en los tres estudios

El método de la canasta normativa alimentaria utilizado en el estudio CEPAL-PNUD

Al procedimiento aplicado en el estudio CEPAL-PNud lo he denominado (Boltvinik, 1990, 1991, 1992a) la variante de la canasta normativa alimentaria (CNA) del método de la línea de pobreza (LP). Consiste en 
los siguientes pasos: $a$ ) se define una canasta normativa de alimentos y su costo es interpretado como la línea de pobreza extrema o de indigencia per cápita; $b$ ) a continuación se divide tal línea entre el coeficiente de Engel (proporción del gasto del hogar destinado a la alimentación) para obtener lo que se denomina la línea de pobreza per cápita; c) las dos líneas de pobreza per cápita se comparan con el ingreso per cápita de cada hogar. Los hogares que tienen ingresos per cápita inferiores a la línea de pobreza se consideran pobres. Los que tienen ingresos inferiores a la línea de pobreza extrema o indigencia, se clasifican como pobres extremos. Las personas reciben el atributo del hogar.

Con este método no se mide la pobreza en general, sólo la pobreza alimentaria. El punto crítico del procedimiento es el paso de la línea de "pobreza extrema" (costo de la CNA), a la línea de pobreza. El supuesto implícito, explicitado por Óscar Altimir (1979: 42) es “...que los hogares que se hallan por encima del umbral mínimo de alimentación se hallan también por encima de los umbrales mínimos para otras necesidades básicas".

Como lo han demostrado los trabajos de Beccaria y Minujin (1987) en Argentina, de Kaztman (1989) en Montevideo y los del PRSP-PNUD (1990, 1991, 1991a, 1992a, ) en numerosos países de América Latina, la evidencia empírica demuestra abrumadoramente que éste es un supuesto falso (Boltvinik, 1990). En efecto, muchos hogares no pobres por LP -y por tanto con satisfacción de la necesidad alimentaria-sí lo son por NBI, por lo que la satisfacción de aquélla no supone necesariamente la de vivienda, agua, asistencia escolar de los meno res, etcétera.

Su falsedad, sin embargo, es no sólo empírica, pues el supuesto lleva implícita una concepción particular sobre la satisfacción de las necesidades básicas, en la cual se produce un proceso de acercamiento simultáneo a la satisfacción de todas las necesidades y en la cual no existe variación individual en el orden de su satisfacción. Bastaría con observar una de ellas para saber cuál es la situación de todas las demás.

Sin embargo, con este método se identifica a la población cuyo gasto alimentario per cápita es menor que el costo de la canasta alimentaria, población a la que he calificado en situación de pobreza alimentaria. Por tanto al método de la cNA lo podemos llamar método de medición de la pobreza alimentaria. Aunque debe haber un error en ello, cuya importancia sólo se puede estimar empíricamente, éste es 
mucho menor que el derivado de interpretar los resultados como pobreza en general. ${ }^{3}$

En cuanto a la línea de pobreza extrema, he dicho en otra parte (Boltvinik, 1990 y 1991) que la definición de pobreza extrema como la situación de aquellos hogares que aun dedicando todo su ingreso a alimentos, no podrían satisfacer sus necesidades en la materia, es inaceptable porque ello simplemente no es posible.

Encontrarse bajo la línea de pobreza de la CNA no necesariamente conlleva una nutrición inadecuada. Un individuo, o una familia, podría tener hoy día una dieta similar a la que prevalecería como norma si los patrones de consumo del entorno social no se hubiesen encarecido (como ocurrió en América Latina en los años setenta y ochenta), es decir, con plena satisfacción de los requerimientos nutricionales, pero por abajo de las normas actuales que se basan en dietas con mayor presencia de productos de origen animal, frutas y verduras. Sin embargo, al sostener como lo hago (y como implícitamente lo hace el estudio CEPAL-PNUD) que dicha persona se encuentra en pobreza alimentaria, estoy introduciendo un concepto de alimentación humana ajustado a las pautas socialmente prevalecientes en cada sociedad concreta, tanto en el tiempo como en el espacio. Un concepto relativo de pobreza alimentaria. En los extremos de la pobreza alimentaria, se presentan, sin duda, carencias biológicas, desnutrición, pobreza absoluta. Entonces, la inadecuada nutrición humana es condición suficiente, pero no necesaria, de la pobreza alimentaria.

\section{Los procedimientos de medición de la pobreza del Banco Mundial}

El Banco Mundial (вм, 1990, 1992) en su estudio sobre la pobreza en el mundo, utilizó una línea de pobreza (LP) de 370 dólares per cápita al año, y una línea de pobreza extrema (LPE) de 275, ambas en dólares de paridades de poder adquisitivo (PPA) de 1985. Ello a pesar de que el trabajo muestra que las líneas de pobreza que se utilizan en los países son, en general, una función positiva del ingre-

${ }^{3}$ Lamentablemente el estudio (EPAL-70 (véase Altimir, 1979) incurre en la arbitrariedad de adoptar un mismo coeficiente de Engel (0.5), para todos los países a pesar de la amplia variabilidad mostrada en los datos observados. El estudio CEPAL-PNUD (1990, 1992) cometió la doble arbitrariedad de usar el mismo coeficiente de Engel de CEPAI-70 para todos los países, a pesar, nuevamente, de la evidencia. Con estas arbitrariedades, el uso empírico de los datos se oscurece, aunque el conceptual permanece claro. 
so per cápita de éstos. Un trabajo más reciente (Banco Mundial, 1993), aborda la distribución del ingreso y la pobreza en América Latina durante la década de los ochenta.

El вм deja claro que se trata de un estudio de pobreza absoluta, la cual define "como una medida de los individuos cuyo bienestar es menor que un estándar absoluto". Este estándar absoluto lo define en términos de ingresos solamente a pesar de percatarse que “...el uso de la dimensión única del ingreso como criterio de bienestar deja de tomar en cuenta la importancia de beneficios recibidos de fuentes diferentes...".

El вм adopta una línea de pobreza y otra de pobreza extrema de "iguales poderes adquisitivos", cuyo nivel fija para todos los países arbitrariamente en 60 dólares de paridades de poder adquisitivo (PPA) de 1985, mensuales por persona (1993: 54). La línea de pobreza extrema (LPE) la fija en 30 dólares, suponemos que siguiendo la pauta de CEPAL-PNUD de que ésta sea la mitad de la de pobreza y que, si se alarga la semejanza, debería ser igual al costo de los alimentos, aunque como veremos después, no es el caso.

¿Qué significa la pobreza medida por esta linea arbitraria? Una idea puede obtenerse de los valores de estas líneas en monedas nacionales. Me limitaré a analizar el caso de México donde la línea de pobreza de 60 dólares (PPA de 1985) equivale a un ingreso, en pesos corrientes de ijunio-agosto de 1989 , de 75600 pesos mensuales per cápita. Para una familia de cinco personas el monto requerido sería, por tanto, de 378000 pesos al mes, lo que correspondía a 41.3 días de salario mínimo del D.F. Es decir, si en el hogar promedio de 4.93 personas, en el cual trabajan 1.63 personas -según la ENIGH-89-, todos ellos lo hicieran a cambio de un salario mínimo, el hogar tendría un ingreso de 448000 pesos, casi $20 \%$ arriba de la LP y 2.4 veces la LPE. Los salarios mínimos reales de 1989 eran aproximadamente $50 \%$ de lo que fueron en el periodo 1976-1981 (en otros términos, los salarios de este periodo eran el doble de los de 1989) por lo que en ese periodo nuestra familia se situaba a 2.4 veces la $L P$ y a 4.8 veces la $L P E$ $\operatorname{del} B M$.

En términos de la Canasta Normativa de Satisfactores Esenciales (CNSE) que desarrollé como parte de los trabajos de Coplamar (Boltvinik, 1984), la LP del Banco Mundial representa $28.9 \%$ de su costo, y la de pobreza extrema $14.5 \%$. La LPE del вм representa $76.5 \%$ de la canasta de alimentos Coplamar 1 (Coplamar, 1982), y sólo $41.2 \%$ de la de CEPAL-PNUd. La LP del Banco Mundial, que es el doble de la LPE, permite adquirir, por tanto, $82.4 \%$ de la canasta alimentaria de CEPAL- 
PNUD, si se dedica todo el ingreso a alimentos. La LP del вм es más baja que la LPE de CEPAL-PNUD. ¿Qué significado tiene entonces la pobreza que mide el вм?

Tomemos en cuenta la enorme variabilidad de soluciones de dietas con cobertura de los requerimientos nutricionales. La canasta alimentaria Coplamar 1 tiene un costo de 54\% de la de CEPAL-PNud (Boltvinik, 1992). Aquella canasta, es necesario aclararlo, contiene sólo 34 alimentos, no contiene ninguna bebida ni lácteos (excepto leche), y no contiene alimentos consumidos fuera del hogar. La línea de pobreza del вм representa $152.7 \%$ del costo de dicha canasta, lo que significa que hogares que dediquen $65.5 \%$ de su ingreso a alimentos crudos, podrían con un ingreso igual a tal LP, adquirir la canasta Coplamar 1. Los datos disponibles de la Encuesta Nacional de Ingresos y Gastos de 1989 (ENIGH-89) muestran que el coeficiente de Engel del gasto monetario resulta ser de $56.3 \%$ en el decil I (el más pobre); $52 \%$ en el decil II y sigue disminuyendo de ahí en adelante. Es decir, que la LP del Banco Mundial no mediría siquiera la pobreza alimentaria ni aun con base en una canasta alimentaria muchísimo más barata y reducida que la de CEPAL-PNUD.

Desde luego, es posible conformar canastas todavía más baratas que la Coplamar 1. Esta está construida con base en los hábitos alimentarios del decil $\mathrm{V}$ nacional de la encuesta de ingresos y gastos de 1977. Esto sería demasiado caro para el вм, ya que de acuerdo con éste (1990: 30, cursivas nuestras): "el costo de adquirir alimentos suficientes para una ingestión calórica minima... puede calcularse examinando los precios de los alimentos que constituyen la dieta de los pobres". Nótese que el BM reduce los requerimientos nutricionales a calorías, en contra de las recomendaciones de la FAO/oms/onv. La dieta del decil más pobre, el I nacional, comprende sólo $15.4 \%$ del gasto total en carnes, contra $34 \%$ en la canasta Coplamar 1. La línea de pobreza del Banco Mundial se podria interpretar como una línea de desnutrición o linea de sobrevivencia físi$c a$. Es posible que reduciendo el costo de la canasta alimentaria más abajo de la Coplamar 1, la LP del BM.permitiera que una persona con ese ingreso, y dado su coeficiente de Engel, adquiriera esa dieta de pobres para cubrir sus requerimientos calóricos. Naturalmente, si las demás necesidades quedan en un umbral de incertidumbre con la línea CEPAL-PNUD, puede afirmarse, sin temor a equivocarse, que con la canasta del Banco Mundial todas las demás necesidades quedan insatisfechas. Naturalmente, la LPE del Banco no tiene ningún sentido y las personas con ese nivel de ingresos estarían técnicamente muertas. 
El método de NBI-LP usado por el Proyecto Regional de Pobreza del PNUD

Siguiendo una línea analítica iniciada por Beccaria y Minujin (1987) y por Kaztman (1989), y una postura conceptual que yo señalara por primera vez (Boltvinik, 1990, 1990a) en el sentido de que el uso simultáneo de los procedimientos de NBI y de LP constituye un nuevo método, el PRSP-PNUD impulsó y llevó a cabo la medición de la pobreza por este nuevo método.

El método NBI-LP consiste en el uso simultáneo, pero acrítico, de los métodos de LP y de NBI tal como se venían usando en la práctica latinoamericana, es decir, la variante de Canasta Normativa Alimentaria (CNA) y el procedimiento conocido como NBI, cuyo prototipo es el trabajo La pobreza en Argentina (INDEC, 1984). Este método ha sido criticado en detalle en Boltvinik (1994a y 1994b). Es necesario distinguir este procedimiento tradicional de NBI de la versión mejorada, que he desarrollado como componente del Método de Medición Integrada de la Pobreza (ммір). ${ }^{4}$

El criterio de pobreza adoptado en el método consiste en considerar. como pobres aquellos hogares (y sus ocupantes) cuyo ingreso per cápita se encuentre por abajo de la línea de pobreza per cápita y/o tenga una o más necesidades básicas insatisfechas. Es decir, se consideran pobres los hogares que se encuentran en la unión de ambos conjuntos. Con esto el total de pobreza encontrada en cualquier país se eleva, a veces considerablemente, respecto a lo encontrado por cualquiera de los dos métodos que integra. Cuatro categorías resultan de la combinación de ambos métodos: a) los pobres por ambos métodos (la intersección de los conjuntos); b) los pobres por LP y no pobres según NBI; c) los pobres por NBI y no pobres por LP, y $d$ ) los no pobres por ambos métodos.

En la crítica del método NBI-LP se pueden distinguir los siguientes elementos: a) el análisis crítico de los dos métodos parciales que integra; $b$ ) la discusión de la manera en que logra dicha integración; $c$ ) la discusión del criterio de pobreza.

Los dos métodos se integran por el expediente simple de aplicar ambos al mismo universo de hogares y aplicarles el criterio de pobreza arriba descrito. Esto, sin embargo, equivale a una integración mecánica,

${ }^{4}$ Para la definición operacional de la versión mejorada de NBI, así como los resultados de su aplicación al censo de 1992, véase Udapso (1994), así como Boltvinik (1994 y 1994b). 
puesto que ni siquiera se analiza la posible existencia de duplicidades. Una de ellas resulta evidente: el indicador indirecto de ingresos usado en el método de NBI (que se construye como un indicador compuesto del nivel educativo delijefe del hogar y la tasa de dependencia económica del mismo) duplica el de ingresos de t.p. Otra muestra del carácter mecánico de la integración radica en el hecho de que la línea de pobreza no se sujeta a revisión alguna, cuando es evidente que algunos rubros identificados por NBI no deben incluirse en la línea de pobreza, porque ello equivale a verificarlos nuevamente. Es el caso de la vivienda.

Por último, en referencia al criterio de pobreza, no es evidente que un hogar deba ser considerado pobre si tiene alguna necesidad insatisfecha o se encuentra por debajo de la línea de pobreza. En Boltvinik (1992a) exploré sistemáticamente esta pregunta. En la intersección de los conjuntos la duda no se presenta. Es sólo en los casos de las pobrezas parciales (identificada solamente en una de las dos dimensiones) que surge. Una manera de hacer evidente las razones de esta duda es llevando la situación de no pobreza en una de las dimensiones al extremo. Si el hogar es no sólo no pobre por ingresos, sino millonario, parecería que la presencia de una NBI, digamos la no asistencia escolar de uno de los niños, no calificaría al hogar como pobre; igualmente, la pobreza por ingresos -sobre todo en la medida que el periodo de referencia para captarlos sea más corto, y que la variable utilizada sea ingresos y no consumo- no necesariamente nos hace concluir que el hogar es pobre, puesto que los hogares ricos pueden vivir muy bien durante años "comiéndose sus ahorros" con ingresos iguales a cero.

\section{La magnitud de la pobreza en América Latina según los tres estudios}

En el cuadro 1 se presentan las cifras de incidencia de la pobreza en América Latina según los tres estudios. Lo primero que resalta es la muy diversa incidencia de la pobreza entre ellos. Mientras el del Banco Mundial identifica a menos de la tercera parte de la población de América Latina como pobre (31.5\% en 1989), el de cEPAL-PNud sitúa la cifra en un poco menos de la mitad (47\% en 1990), y el PRSP-PNUD identifica más de $60 \%$ (61.8\% en 1990). Este último porcentaje es casi el doble que el del вм. En términos absolutos la cifra mínima, la del вм arroja 133 millones de personas; la intermedia, de CEPAL-PNUD, 203 
millones, y la del proyecto, la más alta, 271 millones. Más de $130 \mathrm{mi}-$ llones separan los dos extremos. Como vimos, la del вм puede interpretarse como una línea de sobrevivencia física (o de pobreza alimentaria absoluta) y la de cePAL-PNud como una línea de pobreza alimentaria relativa. La del proyecto estaría más cerca de una imagen integral de la pobreza, en la cual, sin embargo, hay factores de subestimación (el concepto limitado con el que se construye la línea de pobreza y el nivel muy poco exigente de algunos indicadores de NBI) y otros de sobrestimación (el criterio de pobreza, que identifica la unión de ambos conjuntos como pobres), cuyo balance sólo podrá conocerse a medida que se avance en el trabajo empírico en el MMIP.

CUADRO 1

Incidencia de la pobreza en América Latina según tres organismos

\begin{tabular}{|c|c|c|c|c|c|c|}
\hline \multirow{2}{*}{$\frac{A \tilde{n} o}{1970}$} & \multicolumn{2}{|l|}{$\begin{array}{l}C E P A L \\
L P(C N A)\end{array}$} & \multicolumn{2}{|c|}{$\begin{array}{l}\text { Banco Mundial } \\
L P=60 \text { dol.ppa }\end{array}$} & \multicolumn{2}{|c|}{$\begin{array}{l}P R S P-P N U D \\
L P-N B I \\
\end{array}$} \\
\hline & $47.0 \%$ & $130 \mathrm{~m}$ & & & & \\
\hline 1980 & $41.0 \%$ & $144 \mathrm{~m}$ & $26.5 \%$ & $91 \mathrm{~m}$ & & \\
\hline 1986 & $43.5 \%$ & $175 \mathrm{~m}$ & & & $61.5 \%$ & 248 \\
\hline 1989 & & & $31.5 \%$ & $133 \mathrm{~m}$ & & \\
\hline 1990 & $47.0 \% *$ & $203 \mathrm{~m}$ & & & $61.8 \%$ & 271 \\
\hline 2000 & $44.0 \% *$ & $232 \mathrm{~m}$ & & & $56.0 \%$ & 296 \\
\hline Incr. & $70-80$ & $14 \mathrm{~m}$ & $80-89$ & $42 \mathrm{~m}$ & & \\
\hline & $80-90$ & $59 \mathrm{~m}$ & & & & \\
\hline Inc. & $70-80$ & $18.4 \%$ & $80-89$ & $54 \%$ & & \\
\hline Marg. & $80-90$ & $70.2 \%$ & & & & \\
\hline
\end{tabular}

* Proyecciones del PRSP-PNUD.

A pesar de las diferencias en los conceptos de pobreza (que se reflejan en las líneas de pobreza dispares utilizadas) y, en consecuencia, de las muy contrastantes incidencias que identifican, ambos estudios coinciden dramáticamente en un aspecto: el fuerte incremento de la pobreza en la década perdida. La proporción de personas en pobreza alimentaria relativa pasó, según CEPAl, de $41 \%$ en 1980 a $47 \%$ en 1990 (un incremento de 6 puntos porcentuales que representa alrededor de $15 \%$ en relación con el porcentaje inicial) y que supone la vuelta a los niveles de 1970; la proporción de personas en pobreza alimentaria absoluta, según el вм, pasó de 26.5 a $31.5 \%$ (un incremento de 5 puntos porcentuales, que representa $19 \%$ respecto al nivel inicial). 
En ambos casos se trata, como se ve, de aumentos porcentuales muy importantes, aunque en términos relativos resulta mayor el identificado por el вм, especialmente si se toma en cuenta que se refiere a un periodo de nueve años contra diez de cEpal.

En términos absolutos, el incremento de pobres alimentarios relativos fue de 59 millones, mientras el de pobres alimentarios absolutos habría sido de 42 millones. Expresando ambos incrementos en relación con el incremento poblacional ocurrido en el periodo, obtenemos la incidencia marginal de la pobreza, que nos dice qué proporción se encuentra en pobreza del total de habitantes que se añadieron al stock poblacional. Esta incidencia marginal resulta de $70.2 \%$ y de $54 \%$, en los casos relativo y absoluto de la pobreza alimentaria, lo que muestra la gravedad del proceso ocurrido. Si relacionamos esta incidencia marginal con la incidencia media de 1980 , confirmamos una vez más que la pauperización que capta el estudio del Banco Mundial es más acelerada que la que capta CEPAL-PNUd: en el primer caso la relación es 2.04 y en el segundo es 1.72 . Si diéramos a estos estudios la categoría de rigurosos -el de cEPAL-PNUd está menos lejos de serlo que el del Bм- y tomáramos, por tanto, sus resultados como reflejo de los cambios ocurridos en la realidad, concluiríamos que el proceso de pauperización acelerado de los ochenta, lo fue más en los estratos de la pobreza más aguda.

\section{Las políticas de lucha contra la pobreza de los tres organismos}

\section{Rasgos generales}

Para el Banco Mundial la lucha contra la pobreza es el elemento que viene a complementar sus políticas de ajuste estructural. Las propuestas en materia de política de lucha contra la pobreza se insertan plenamente en lo que se ha llamado el Nuevo Modelo Económico o modelo neoliberal (de aquí en adelante NME), del cual el вм es uno de sus líderes y promotores más destacados. De hecho uno de los cuatro principios básicos del NME es:justamente el compromiso de lucha contra la pobreza absoluta. Los otros tres son: 1) el nuevo y más reducido papel del Estado en favor del creciente rol de las fuerzas del mercado; 2) la altísima prioridad de la estabilidad macroeconómica -mantenimiento de los equilibrios macroeconómicos básicos: baja inflación, equilibrio presupuestal y equilibrio externo-, y 3) una mayor apertura 
de la economía al comercio internacional y a los flujos internacionales de capital privado (véase Scott, 1994). El BM adopta el paquete completo del NME y, por tanto, en materia de pobreza propone sólo medidas que no contradigan los otros tres pilares. Por tanto, evita cualquier acción que interfiera con las fuerzas del mercado, porque ello, de acuerdo con la teoría económica estándar -o neoclásica-, lleva a ineficiencias en la asignación de recursos. La inclusión de la lucha contra la pobreza viene del reconocimiento de los seguidores de estas ideas, que el mercado es incapaz de resolver eficientemente algunos aspectos del desarrollo como la formación de recursos humanos o el cuidado de la salud, y de una evidente -aunque no siempre explícita- preocupación por la estabilidad política.

La cEPAL formula una estrategia de desarrollo que denomina "enfoque integrado" en la cual se plantea avanzar simultáneamente en el crecimien to económico y la equidad. Rechaza, para ello, la visión que plantea la necesidad de crecer primero para distribuir después, asî como la postura que supone que a la política económica toca la promoción del crecimiento y a la social el tema de la distribución, para arribar a la conclusión de que no puede "generarse crecimiento con equidad sin que ambos constituyan objetivos tanto de la política económica como de la política social" (CEPAL, 1992: 16). Esta propuesta no utiliza, a diferencia de las otras dos, de manera central el término de pobreza sino el de equidad (que más adelante se discute su significado). Aunque rebasa los límites de este ensayo probarlo, me parece que cepal parte de la aceptación de la política económica del NME, con algunos matices, particularmente porque percibe la necesidad de un Estado más promotor y en algunos casos más interventor. Este marco de política económica se verá matizado, además, para alcanzar la equidad, por el enfoque in tegrado que implica "por una parte, preferir aquellas políticas económicas que favorecen no sólo el crecimiento, sino también la equidad, y por otra, destacar en la política social el efecto productivo y de eficiencia, y no sólo de equidad" (CEPAL, 1992: 16, cursivas nuestras).

El planteamien to del PRSP-PNUD de "desarrollo sin pobreza" supone, desde el diagnóstico y la medición, un carácter integrado. Por ejemplo: "Es necesario atacar, con diferencias de énfasis relativo en cada país, las tres raíces conceptuales de la pobreza: la insuficiencia del excedente generado, su apropiación inequitativa, y las inadecuaciones en la estructura de la oferta y de la demanda de ciertos satisfactores" (PRSP-PNUD, 1990: 101). La superación de la pobreza se convierte en el objetivo central del desarrollo, apoyándose en las mediciones realizadas: 
"En tanto más de la mitad de la población latinoamericana es actualmente pobre y la mayor parte de ella tiene un origen estructural, la superación de la pobreza no puede seguirse viendo como un programa o como una tarea parcial a cargo de la política social sino que debe concebirse como objetivo central del desarrollo" (PRSP-PNUD, 1990: 102, cursivas en el original). Aunque asume básicamente como dado el marco de política macroeconómica, sugiere un cierto grado de heterodoxia: "En la transición a la estabilidad, sin embargo, la combinación de una política macroeconómica ortodoxa con mecanismos 'heterodoxos' de concertación y fijación de precios básicos parece la mejor opción" (PRSP-PNUD, 1990: 118).

Las raices de la pobreza seguin los tres organismos

El Banco Mundial sostiene, acudiendo a un análisis contrafactual, que la evolución de la pobreza hubiese sido peor si las reformas económicas no se hubiesen puesto en práctica, lo cual se comprobaría por el hecho de que los países que llevaron a cabo las reformas requeridas tuvieron un mejor desempeño que los que no las efectuaron (вм, 1993: IX у вм et al., 1993b: ii).

Sin embargo, el análisis contractual que realiza la institución no cumple con los requisitos científicos requeridos, ya que se impondría que el propio Banco Mundial, el FMI y otras instituciones internacionales hubiesen otorgado el mismo trato a los países que hicieron las reformas y a los que no las hicieron. De otra manera es imposible separar el efecto de las reformas (o de su ausencia) de los efectos de los premios por haberlas llevado a cabo (o castigos por no haberlas realizado).

La visión de las raíces de la pobreza parcialmente explícita en el discurso del вм, es la de atribuir al menos una parte importante de ella al manejo incorrecto de la política macroeconómica. Los casi 40 años de sustitución de importaciones en la región habrían sido un error, pues se interfirió con la libre competencia internacional y, por tanto, no se lograron desarrollar los campos en los que los países tienen ventajas comparativas, manteniendo graves distorsiones que bajaron mucho la eficiencia en la asignación de recursos; el Estado intervino demasiado, tan to como empresario como en la formación de precios. Por ejemplo, la legislación de los salarios mínimos impediría el aprovechamiento pleno de la fuerza de trabajo porque mantendría arti- 
ficialmente altos los salarios; lo mismo se puede decir respecto del papel de los sindicatos. El manejo inadecuado de la política macroeconómica habría llevado también a periodos inflacionarios importantes que serían los causantes de buena parte de la pobreza.

El trabajo de CEPAI donde se expone el enfoque integrado dedica un capítulo a analizar las condiciones sociales de la región, mismo que titula "Los rezagos en materia de equidad". En él se constata que las tasas de fecundidad de la población pobre son más altas que las de los no pobres. Y que los países más pobres registran tasas de crecimiento poblacional más alto que los menos pobres. De aquí, sin embargo, pasa a una tesis malthusiana notable y lamentable: “...se estimó que en Guatemala $98 \%$ de la cantidad adicional de pobres e indigentes surgida durante la década se atribuiría al crecimiento natural de la población en tales condiciones, porcentaje que sólo alcanzaría a $55 \%$ en Costa Rica. Esto significa que en el caso del último país otros factores socioeconómicos, y no sólo los demográficos, explicarian el aumento de la pobreza" (CEPAL, 1992: 33, Recuadro 1-1, cursivas nuestras). Naturalmente, después de tan sabia tesis, se seguiría que la única política para evitar el crecimiento de la pobreza es el control natal, al menos en Guatemala, y todo el resto del libro de la cEPAL saldría sobrando, así como el resto del análisis del capítulo que nos scupa, que pretende relacionar la crisis con los movimientos de la pobreza. El planteamiento es casi racista: hijo de madre pobre nace pobre y pobre se queda. La pobreza es genética y se hereda. Es notable que este texto exista y que en el resto del capítulo se actúe como si no existiera. Es obvio que la dinámica demográfica consiste no sólo en nacimientos y muertes, sino en la formación y disolución de hogares. La población crece básicamente a través del crecimiento en el número de hogares, ya que el tamaño promedio de los hogares es bastante estable y con una tendencia a disminuir lentamente. Por tanto, suponiendo que los hogares preexistentes se conserven y mantengan su estatus de pobreza-no pobreza, el punto decisivo en términos del crecimien to de la pobreza resultaría ser el estatus de los nuevos hogares, de los nuevos matrimonios. Esto, naturalmente, depende fundamentalmente, aunque no exclusivamente, de la inserción ocupacional de los adultos activos de estos hogares. Y esto es un fenómeno económico y no demográfico. La contabilidad absurda de CEPAL haría que toda la explicación de la pobreza en el mundo se pudiera explicar por el empobrecimiento de Caín -dadas sus malas costumbres-y la alta tasa de fecundidad de él y sus descendientes. Es incompatible con periodos de baja en la pobreza 
f) En materia de gasto social -salvo las tareas asistenciales cuya ausencia pondría en riesgo la sobrevivencia- debe otorgarse preferencia a las políticas sociales funcionales al desarrollo y las orientadas a romper el círculo vicioso de la pobreza, como la paternidad responsable, los programas de atención materno-infantil, la educación preescolar y los programas de capacitación de los trabajadores. El sistema de seguridad social debe ampliarse en su cobertura. Deben canalizarse bonificaciones al salario de los jefes de hogar con más bajos ingresos.

El planteamiento se enraiza profundamente en el Nuevo Modelo Económico pero a la vez intenta separarse de él. Muchas de las propuestas están en la discusión pública desde hace mucho, como la reforma laboral, o la creación o simulación de mercados que es una de las modas del pensamiento económico, del llamado nuevo institucionalismo. Sin embargo, la CEPAL las envuelve en un halo de novedad. Lo que resulta evidente es que la CEPAL carece de una respuesta a los problemas básicos de la pobreza, que su propio diagnóstico puso de relieve.

La fragilidad de la propuesta radica en que está apoyada en varias apuestas -no se pueden llamar de otra manera ya que la evidencia aportada por la CEPAL es muy escasa- orientadas a restarle importancia a tres aspectos contradictorios del desarrollo capitalista: campo técnico y empleo; nivel de salarios y de la tasa de ganancia; distribución del ingreso y ahorro.

La CEPAL no enfrenta el hecho, destacado en su propio diagnóstico, que los salarios se han deteriorado bruscamente en la mayoría de los países y que ello constituye la causa inmediata básica del aumento en la pobreza en los años ochenta. A esto se aúna la postura ambigua de la CEPAL respecto de la pequeña producción popular, reflejada desde el diagnóstico, lo que le lleva a no estructurar una propuesta cabal para su desarrollo (solamente aborda el tema del financiamiento a la pequeña y mediana empresa, aparentemente dejando fuera la popular). Sin un planteamiento para los salarios y para la pequeña producción popular (dos elementos fundamentales de la masa de ingresos populares), la disminución de la pobreza queda librada a que los mecanismos del mercado, las reformas laborales, los salarios participativos, generen los empleos formales que el propio volumen inicial de Transformación productiva con equidad (cE.PAL, 1990: 81) preveía que no se podrían generar. En contraste el PRSP-PNUD ha estructurado detalladamente una estrategia para su apoyo en el volumen La economia popular. Una via para el desarrollo sin pobreza en América Latina (PRSP-PNUD, 1991). 
En cuanto a las propuestas del Banco, el componente de servicios sociales termina siendo el central para esa institución y conviene analizarlo con algún detenimiento. El desarrollo de la propuesta se encuentra en el capítulo 5 del informe de 1990. En él se reitera la tesis del capital humano:

Se invierte muy poco en su capital humano [de los pobres], lo cual aumenta la probabilidad que ellos y sus hijos sigan siendo pobres. Para romper este círculo vicioso, los gobiernos deben hacer que el objetivo de que los servicios lleguen a los pobres sea una prioridad por derecho propio (p. 79).

El Banco recomienda promover la participación del sector privado en educación y en salud como una manera de permitir que el sector público dirija una mayor parte de sus recursos hacia los pobres que no pueden pagar por los servicios privados. Igualmente, recomienda seguir el ejemplo chileno de promover la prestación privada de servicios de educación con financiamiento público y obligar a todas las escuelas, públicas y privadas, a competir por los alumnos, pagando los subsidios únicamente en función de los alumnos atendidos.

En materia de salud, el Banco parte de que "los argumentos a favor de que el Estado sea el único proveedor de asistencia curativa carecen de fuerza”. El Banco propone entonces un sistema de salud casi enteramente privado, con un rol subsidiario para el Estado que deberá cubrir el campo de la salud pública en las regiones aisladas o sin atractivos de mercado, y la atención básica a los pobres. Aunque hay pasajes del mismo texto en que esto se contradice y parece sostenerse que el sector público seguirá siendo el principal proveedor $\mathrm{y} / \mathrm{o}$ fmanciador, siendo las reformas del Chile de Pinochet el ejemplo favorito. Tanto para la educación como para la salud, el BM recomienda "reorientar la asignación de recursos" de los servicios de nivel superior en favor de la educación primaria y la atención básica de la salud, con lo cual se logrará "una mayor eficacia y equidad" (Banco Mundial, 1990). El organismo financiero insiste en que se debe cobrar a los usuarios. A pesar de que advierte que "según datos recientes, la demanda de asistencia médica es sensible al costo, y más en el caso de los pobres que de los ricos", y que eso significa "que si se cobran honorarios (o se aumentan) los pobres serán los primeros en desertar", de ahí concluye que los servicios no deberían ser enteramente gratuitos y que a "veces podrían fijarse tarifas nominales en las zonas pobres" y que "debería considerarse la posibilidad de cobrar honorarios en el caso de los ser- 
vicios (en especial los hospitalarios) que rinden mayormente beneficios individuales" (cursivas nuestras).

$\mathrm{Al}$ enunciar los principios sobre la salud quedan clarísimas las bases doctrinarias de toda la propuesta. Primero, el principio de subsidiariedad del Estado de acuerdo con el cual, según explica Pilar Vergara (1990: 37), "éste debe abstenerse de toda forma de intervención y asumir únicamente aquellas responsabilidades que los particulares o las organizaciones sociales no están en condiciones de desempeñar adecuadamente". Segundo, se reduce el campo de los servicios sociales a educación y salud, dejando fuera notablemente la vivienda y sus servicios. Aunque ello no significa que los ignore del todo, es evidente que el acento está puesto en otra parte. El enfoque de capital humano suele "olvidar" necesidades básicas tan fundamentales como la vivienda, y parecería suponer seres humanos que comen, cuidan su salud y se educan en un vacío espacial, por lo cual la vivienda no resulta prioritaria. En agudo contraste, algunos movimientos populares de la ciudad de México, como la Asamblea de Barrios, marchan por las calle gritando: "que el gobierno entienda, lo primero es la vivienda". Tercero, para el Banco sólo los beneficios apropiados colectivamente merecen subsidio del Estado, lo cual refleja que su preocupación por los pobres está teñida con su preocupación por los efectos externos negativos de la pobreza en los no pobres. Sería gravísimo así que un rico enfermase por contagio de un pobre. En este caso, es obligación del Estado intervenir y subsidiar $100 \%$. Pero si un pobre enferma de cáncer en la próstata debe cobrársele la atención, así sea pobre. Estos sesgos están asociados a la insistencia en la supuesta prioridad de los servicios primarios o básicos de salud, que es en donde se suelen atender las enfermedades infecciosas o contagiosas. En cambio, las crónicas o degenerativas, cuya asistencia sólo beneficia al paciente, y que suelen ser materia del tercer nivel de atención, no resultan prioritarias. En los países de América Latina donde se vive plenamente la transición epidemiológica, no puede sostenerse que los pobres sólo se enferman de padecimientos atendibles en el primer nivel.

Por último, frente a las propuestas del Banco Mundial de estimular la inversión privada en educación como una manera de permitir al Estado concentrar sus esfuerzos en los más pobres cabe advertir una aguda observación de la cEPAl que: "...en la medida en que se deteriore la calidad de la educación pública y los estratos de mayores ingresos prefieran la opción privada, tenderán a consolidarse circuitos estratificados de educación, trabajo y bienestar" (1992: 42). 


\section{Bibliografía}

Altimir, Óscar (1979), La dimensión de la pobreza en América Latina, Santiago de Chile, cEPAL (Cuadernos de la CePAL, 27).

вм (Banco Mundial) (1990 у 1992), La pobreza. Informe sobre el desarrollo mundial 1990, Washington. [Las partes centrales del capítulo 2 fueron reproducidas en Comercio Exterior, vol. 42, núm. 4, 1992.]

(1993), Poverty and Income Distribution in Latin America. The Story of the 1980s, Washington.

(1993a), Poverty Reduction Handbook, Washington.

(1993b), C. O. N. Moser., A. J. Herbert, R. E. Makonnen, Urban Poverty in the Context of Structural Adjustment. Recent Evidence and Policy Responses, documento de discusión de rwu, Washington, mayo.

(1993c), Implementing the World Bank's Strategy to Reduce Poverty. Progress and Challenges, Washington.

(1993d), World Development Report, 1993: Investing in Health, Oxford, Oxford University Press.

Barreiros, Lidia (1987), "La pobreza y los patrones de consumo de los hogares", en Lidia Barreiros, Arend Kouwenaar, Rudolf Teekens y Rob Vos, Ecuador. Teoria y diseño de políticas para la satisfacción de las necesidades básicas, La Haya, Instituto de Estudios Sociales de La Haya/Organización Internacional del Trabajo, La Haya. [Reproducido en Comercio Exterior, vol. 42 , núm. 4 , 1992.]

Beccaria, Luis y Alberto Minujin (1987), "Métodos alternativos para medir la evolución del tamaño de la pobreza", documento de trabajo núm. 6, Buenos Aires, Instituto Nacional de Estadística y Censos.

___ y Alberto Minujin (1991), "Sobre la medición de la pobreza: enseñanzas a partir de la experiencia argentina", documento de trabajo núm. 8, Argentina, Unicef, noviembre.

__ Julio Boltvinik, Óscar Fresneda, Amartya Sen et al. (1992), América Latina: el reto de la pobreza, Bogotá, Proyecto Regional para la Superación de la Pobreza (PRSP), PNUD.

Boltvinik, Julio (1984), "Satisfacción desigual de las necesidades esenciales en México", en Rolando Cordera y Carlos Tello (coords.), La desigualdad en México, México, Siglo XXI, pp. 17-64.

(1986), "Modo de producción estatal y satisfacción de necesidades esenciales en México", Investigación Económica, núm. 177, pp. 195-244.

(1986a), "Sistema de necesidades y modo de vida en México", Investigación Económica, núm. 175, pp. 169-204.

(1990), Pobreza y necesidades básicas. Conceptos y métodos de medición, Caracas, PRSP, PNUD.

- (1990a), "Hacia una estrategia para la superación de la pobreza", Necesidades básicas y desarrollo, La Paz, ILPES/ILDIs/Instituto de Estudios Sociales de La Haya, pp. 25-50. 
(1991), "La medición de la pobreza en América Latina", Comercio Exterior, vol. 41 , núm. 5 , pp. 423-428.

- (1992), "Pobreza alimentaria en América Latina”, Archivos Latinoamericanos de Nutrición, vol. 42, núm. 4, pp. 116-125.

(1992a), "El método de medición integrada de la pobreza. Una propuesta para su desarrollo", Comercio Exterior, vol. 42, núm. 4, pp. 354-365.

(1992b), "Metodología para el mapa de pobreza de Bolivia. Informe al Gobierno de Bolivia" (inédito).

(1993), "Mediciones de pobreza e indicadores alternativos de desarrollo", Estudios Sociológicos, vol. 12, núm. 33.

(1994), "Poverty Measurement and Alternative Indicators of Development", en Rolph van der Hoeven y Richard Anker (eds.), Poverty Monitoring. An International Concern, Londres, MacMillan, pp. 57-83.

(1994a), "La pobreza en América Latina. Análisis crítico de tres estudios", Frontera Norte, vol. 6 (número especial: Pobreza), pp. 31-60.

(1994b) Pobreza y estratificación social en México, México, inegr/Colegio de México/insunam.

CEPAL (1990), Transformación productiva con equidad, Santiago de Chile.

(1992), Equidad y transformación productiva: un enfoque integrado, Santiago de Chile.

CEPAL-UNESCO (1992), Educación y conocimiento: eje de la transformación productiva con equidad, Santiago de Chile.

CEPAL-FAo, División Agrícola Conjunta (1988), "Sistemas alimentarios: estructura, evolución y lincamientos de una política de seguridad alimentaria”, LC/R.666, Santiago de Chile.

CEPAL-PNUd (1990 y 1992), "Magnitud de la pobreza en América Latina en los años ochenta", Santiago de Chile, 1990. [En Comercio Exterior, vol. 42, núm. 4, pp. 340-353, se reproducen los capítulos I y II de este trabajo, con el título "Procedimientos para medir la pobreza en América Latina con el método de línea de pobreza".]

Coplamar (1982), Alimentación, México, Siglo XXI, (Serie Necesidades Esenciales en México).

- (1982a), Vivienda, México, Siglo XXI, (Serie Necesidades Esenciales en México).

(1983), Macroeconomía de las necesidades esenciales en México, México, Siglo XXI.

Desai, Meghnad, Amartya Sen y Julio Boltvinik, (1992), Social Progress Index. A Proposal, PNuD, Bogotá [existe también versión en español con las mismas características].

Hobsbawm, Eric (1968), "Poverty", International Encyclopedia of the Social Sciences, Nueva York.

Instituto Nacional del Consumidor (1989), "El gasto alimentario de la población de escasos recursos de la ciudad de México", Comercio Exterior, vol. 39, núm. 1, pp. 52-58. 
INDEC (Instituto Nacional de Estadística y Censos) (1984), La pobreza en Argenti$n a$, Buenos Aires.

Kaztman, Rubén (1989), "La heterogeneidad de la pobreza. El caso de Montevideo", Revista de la CEPAL, núm. 37, pp. 141-152.

Larrea, Carlos (1990), Pobreza, necesidades básicas y desempleo. Área urbana del Ecuador, Quito, Instituto Nacional del Empleo-Instituto Latinoamericano de Investigaciones Sociales.

Mack, Joanna y Stewart Lansley (1985), Poor Britain, Londres, George Alien \& Unwin.

Markus, Giorgy (1973), Marxismo y "antropología", Barcelona, Grijalbo.

Marx, Carlos (1857), "Introducción general a la crítica de la economía política, en Contribución a la crítica de la economía política, México, Siglo XXI, 1980.

Orshansky, Mollie (1965), "Counting the Poor: Another Look at the Poverty Profile", Social Security Bulletin, vol. 28, núm. 1, pp. 3-29.

PNUD (1990-1994), Informe sobre el desarrollo humano. Los informes de 1990, 1991 y 1992, en español, fueron publicados por Tercer Mundo Editores, Bogotá. El de 1993 lo editó el Centro de Comunicación, Investigación y Documentación entre Europa, España y América Latina (CIDEAL), Madrid. El de 1994 fue publicado por el Fondo de Cultura Económica, México. En inglés lo publica Oxford University Press, Oxford.

PRSP-PNUD (Proyecto Regional para la Superación de la Pobreza en América Latina y el Caribe, PNUD) (1990), Desarrollo sin pobreza, Bogotá.

- (1991), Hacia un desarrollo sin pobreza en América Latina y el Caribe. Memorias de la II Conferencia Regional de Pobreza, Bogotá.

(1991a), Pobreza, violencia, desigualdad: retos para la nueva Colombia, Bogotá. (1991b), Development Without Poverty, Bogotá.

(1992a), "Magnitud y evolución de la pobreza en América Latina", Comercio Exterior, vol. 42, núm. 4, pp. 380-392.

(1992b), "Una estrategia para la superación de la pobreza en América Latina", Comercio Exterior, vol. 42, núm. 5, pp. 454-464.

Scott, C. D. (1994), "El nuevo modelo económico en América Latina y la pobreza rural", ponencia presentada en el Seminario Internacional sobre Nuevos Procesos Rurales en México: Teorías, Estudios de Caso y Perspectivas, INAH-Unam, Taxco, 30 de mayo-3 de junio.

Sen, Amartya (1981), Poverty and Famines. An Essay on Entitlement and Deprivation, Oxford, Clarendon Press. Los tres primeros capítulos están traducidos al español (por J. Boltvinik) en Luis Beccaria, Julio Boltvinik, Amartya Sen, Oscar Fresneda et al. (1992). Igualmente pueden encontrarse los capítulos 2 y 3 en Comercio Exterior, vol. 42, núm. 4, pp. 310-322.

Smith, Adam (1776), La riqueza de las naciones, México, Fondo de Cultura Económica, 1981.

Summers, Robert y Alan Heston (1988), "A New Set of International Comparisons of Real Product and Price Leveis Estimates for 130 Countries, 1950-1985", Review of Income and Wealth, vol. 34, núm. 1, pp. 1-24. 
Terrail, Jean Pier (1977), "Producción de necesidades y necesidades de la produccion", en J. P. Terrail et al, Necesidades y consumo en la sociedad capitalista actual, México, Grijalbo, pp. 13-34.

Townsend, Peter (1979), Poverty in the United Kingdom, Harmondsworth, Penguin.

Udapso (Unidad de Análisis de Políticas Sociales) (1994), Mapa de pobreza de Bolivia. Una guia para la acción, Ministerio de Desarrollo Humano.

Vergara, Pilar (1990), Políticas hacia la extrema pobreza en Chile, 1973/1988, Santiago de Chile, flacso. 